\title{
Educación inclusiva en Colombia y la Región del Caribe colombiano: Estrategias para mostrar'
}

\author{
Inclusive education in Colombia and the Colombian Caribbean Region: \\ Strategies to show
}

Rosario Joaquín Reales Vega²

Juan Carlos Berrocal Durán ${ }^{3}$

\begin{abstract}
RESUMEN
El presente artículo forma parte de los resultado de un proceso de investigación llevado a cabo por el Grupo de Investigación Andrés Bello de la Corporación Universitaria Rafael Núñez CURN, cuyo objetivo general se orientó por mostrar y analizar las estrategias que en Colombia se han diseñado y actualmente se aplican para la Educación Inclusiva desde la perspectiva socio-jurídica de la filiación. De manera específica la intención del análisis se concentró en las
\end{abstract}

\footnotetext{
${ }^{1}$ El presente artículo se deriva del Informe Final del proyecto de investigación denominado "Estrategias para la Educación Inclusiva desde la perspectiva socio-jurídica de la filiación, en la Región Caribe Colombiana" con la dirección del investigador Rosario Joaquín Reales Vega del Programa Trabajo Social Sede Barranquilla de la Corporación Universitaria Rafael Núñez, miembro del Grupo de Investigación Andrés Bello. Este proyecto contó con el apoyo de Juan Carlos Berrocal Durán en calidad de Co-investigador, quien aportó sus saberes y experiencias desde el enfoque jurídico. También participaron estudiantes del Semillero de Investigación Rafael Wenceslao Núñez Moledo los cuales se vincularon al estudio con actividades como recolección de datos, organización y análisis de la información: Ruby Villamizar González, Eliana Moreno Duran, Mariana Márquez Moreno (Programa de Derecho) y Mónica Romero Vega, Joismar Cabarcas Vizcaíno (Programa de Trabajo Social).

${ }^{2}$ Sociólogo, Maestrando en Educación. Especialista en Gestión de Proyectos Educativos (Universidad Simón Bolívar). Miembro del Grupo de Investigación Andrés Bello de la Corporación Universitaria Rafael Núñez, Campus Barranquilla, Docente de los Programas Trabajo Social y Derecho de la Corporación Universitaria Rafael Núñez, Campus Barranquilla. Correo: rosario.reales@curnvirtual.edu.co

3 Abogado. Magister en Derecho Procesal (Universidad Simón Bolívar). Miembro del Grupo de Investigación Andrés Bello (Corporación Universitaria Rafael Núñez). Docente de los Programas de Derecho y Trabajo Social de la Corporación Universitaria Rafael Núñez, Campus Barranquilla. Correo: juan.berrocal@curnvirtual.edu.co
}

Recibido: 24 de abril de 2019. Aprobado: 5 de junio de 2019. 
estrategias de lo que en Colombia se viene desarrollando en materia de inclusión en el sector educativo. Para tal fin se dispuso de una serie de técnicas de recolección de información y se planteó un diseño metodológico orientado por el método inductivo, con un tipo de investigación que retomó el enfoque de los postulados de la teoría crítica.

\title{
Palabras clave:
}

Educación, Educación inclusiva, Filiación, Perspectiva socio-jurídica, Responsabilidad del Estado.

\begin{abstract}
This article is part of the results of a research process carried out by the Andrés Bello Research Group of the Rafael Núñez CURN University Corporation, whose general objective was to show and analyze the strategies that have been designed in Colombia and currently they are applied to Inclusive Education from the socio-legal perspective of filiation. Specifically, the intention of the analysis was focused on the strategies of what is being developed in Colombia in terms of inclusion in the education sector. To this end, a series of techniques for gathering information was available and a methodological design was proposed, guided by the inductive method, with a type of research that took up the approach of the postulates of critical theory.
\end{abstract}

\section{Keywords}

Education, Filiation, Inclusive education, Socio-legal perspective, State responsibility.

\section{Introducción}

La educación en Colombia actualmente se encuentra en una encrucijada que se ha visto desbordada por los acontecimientos recientes ocurridos en Colombia, los cuales hacen pensar en la no existencia de fundamentos adecuados desde lo filosófico, teórico y práctico que hagan concertar a todos los actores sociales en unos preceptos convenientes que posibiliten a atención en materia educativa y jurídica, sin distinciones de ninguna índole para el acceso a la educación y dar respuestas a la situación que hoy permea el Sistema Educativo nacional.

La inclusión como figura jurídica recientemente vinculada a la educación no es que se constituya como salvadora de la crisis al interior del sistema, pero apoya con invaluable aporte a que todos los habitantes del país tengan derecho a una educación 
libre de las actuales prebendas que en el país tienen quienes son considerados "sujetos normales", frente a aquellos que por sus condiciones naturales, económicas, culturales, sociales, religiosas, etcétera, son excluidos en forma flagrante, colocándole dificultades para el acceso a la educación en igualdad de condiciones.

Bajo estas premisas surge el interrogante de esta investigación: ¿Cuáles son las estrategias que se proponen para la Educación Inclusiva desde la perspectiva sociojurídica de la filiación, en la Región Caribe colombiana?

En este artículo la intención general está orientada por indicar las estrategias que en Colombia se han diseñado para la Educación inclusiva desde la perspectiva socio-jurídica de la filiación. En términos específicos: describir el sistema educativo de Colombia en relación con los mecanismos de inclusión, fundamentalmente en la ciudad de Barranquilla, capital de la Región Caribe colombiana; analizar retrospectivamente las directrices del Ministerio de Educación Nacional (MEN) con respecto a los modelos pedagógicos colombianos (Berrocal, 2012); identificar los elementos de la práctica inclusiva en el sistema educativo del Caribe colombiano; proponer un ejercicio con estrategias para la Educación inclusiva desde la perspectiva socio-jurídica de la filiación, en la ciudad de Barranquilla.

Se justifica la presente investigación en la medida en que el país y en especial la Región Caribe colombiana, se encuentran comprometidos para que progresivamente, niños y niñas, jóvenes y todas las personas tengan una mayor participación activa en la vida económica, social, política y cultural de sus comunidades y crear una cultura de paz. En este sentido se retoman las aportaciones de $B$. Lindqvist (Naciones Unidas 1994), citado por el Ministerio de Educación Nacional - MEN (2018):

Todos los niños/as y jóvenes del mundo, con sus fortalezas y debilidades individuales, con sus esperanzas y expectativas, tienen el derecho a la educación. No son los sistemas educativos los que tienen derecho a cierto tipo de niños/as. Es por ello, que es el 
sistema educativo de un país es el que debe ajustarse para satisfacer las necesidades de todos los niños/as y jóvenes (p.1).

Esta investigación realza su pertinencia, por cuanto en la Región Caribe de Colombia y en ciudad de Barranquilla, capital de la Región Caribe, es necesario reflexionar sobre las principales teorías que puedan servir de orientación en la elaboración de políticas públicas, normas y disposiciones que desde una perspectiva jurídica, permitan viabilizar armónicamente los hechos relacionados con la inclusión de ciudadanos al sistema educativo, considerando paralelamente los aspectos subyacentes de filiación. Además, se promueve el desarrollo de acciones contextualizadas, que parten del conocimiento socio-cultural de la Región objeto de estudio y que permitirán ofrecer respuestas sensibles a su realidad actual.

\section{Metodología}

El proceso se orientó por el método cualitativo, con un enfoque que retoma los postulados de la teoría crítica a través de una revisión minuciosa de sus términos, valiéndose de las estrategias adecuadas para la recolección, sistematización y análisis de la información obtenida mediante las siguientes técnicas:

- Recolección y análisis de información relacionada con los planteamientos teóricos de los temas anteriormente referenciados y que fundamentan esta investigación.

- Observación directa a los actores en instituciones educativas seleccionadas previamente, con el consentimiento autorizado para los fines investigativos.

- Entrevistas no estructuradas a los actores integrantes de los Grupos Focales (Focus Group), pertenecientes a la sociedad civil e instituciones educativas seleccionadas.

El procedimiento que se utilizó para la recolección de la información tanto de fuente primaria como secundaria es el siguiente: La información de fuente secundaria consignada en los textos y documentos en los cuales se trató cada uno de los aspectos 
referidos a la educación inclusiva, la Adopción y las teorías que desde una concepción critico social han sido expuestas y que son el fundamento y la esencia de la filosofía de normas jurídicas, fueron sujetos de las explicaciones y conceptualizaciones en fichas de análisis documental, las que posteriormente fueron articuladas en cada uno de los capítulos que conformaron los informes, tanto de avances como final.

La información recolectada de fuente primaria y que respondió a observaciones y entrevistas con Grupos Focales siguió los siguientes pasos:

- Selección de las instituciones de educación que fueron sujetos de observaciones en la ciudad de Barranquilla, capital de la Región Caribe.

-Selección de los Grupos Focales (muestra intencional) que fueron sujetos de entrevistas, en la ciudad de Barranquilla, capital de la Región Caribe.

- Sistematización y análisis de la información que vinculada a la redacción de los informes (avance y final) junto con la información de fuente secundaria.

\section{Resultados y Discusión}

\section{El sistema educativo en términos generales en el panorama internacional y en Colombia. Una mirada hacia la educación inclusiva desde la perspectiva de la filiación global}

A nivel mundial se recomienda mantener y alentar la educación inclusiva desde la perspectiva de la filiación. En tal sentido se han presentado alternativas que pretenden mostrar un panorama a las instituciones educativas para que en forma real y efectiva interioricen los postulados, las "posturas ideológicas y los enfoques educativos en torno a las maneras de abordar las diversidades culturales, sociales e individuales que presentan diferencias relevantes entre regiones y al interior de las mismas" (Opertti, 2015). Según este autor, los cinco sub-temas que fueron objeto del debate se orientaron por: 
(a) Mantener las escuelas especiales y fortalecer su coexistencia con las escuelas comunes.

(b) Las escuelas especiales también han sido, y en cierta medida siguen siendo, el refugio y el lugar de "colocación" de grupos socialmente vulnerables. En tal sentido se trata de prácticas de segregación social en sentidos totalmente opuestos al desarrollo de estrategias educativas orientadas al logro de sociedades más inclusivas.

(c) El reconocimiento que los modelos clásicos de integración han posibilitado más bien la incorporación en los niveles de infraestructura y de equipamientos más que en relación a desarrollar propuestas curriculares que enfaticen la diversidad de enfoques pedagógicos y de las prácticas de enseñanza para poder responder a las múltiples expectativas y necesidades de aprendizaje de alumnas/alumnos.

(d) Es más costosa la educación inclusiva en comparación a los modelos tradicionales de educación especial y de integración. Una educación inclusiva se caracteriza por responder a las expectativas y necesidades de todas y todos e implica superar la idea de la segregación / especialización, y de la integración / incorporación. La comparación de los 3 costos no solo debe circunscribirse a la estimación de aspectos vinculados a condiciones y procesos de aprendizaje, sino también involucrar la valoración de los resultados en términos de lograr una educación equitativa de calidad.

(e) La voluntad de buscar superar el debate integración / inclusión a través de la generación de visiones y prácticas inclusivas en los diversos tipos de escuelas. Toda escuela debe asumir un enfoque inclusivo que contribuya a generar condiciones para el logro de sociedades más inclusivas (p.p. 3-4). 
De acuerdo con este escenario en el plano internacional, la figura jurídica de la filiación adquirió la connotación y la moral internacional de hacer válida la idea de igualar tanto a los hijos nacidos dentro como fuera del matrimonio, la cual se consagró y constituye un aspecto regulativo del derecho internacional general; es una regla de lus Cogens, un principio imperativo. En tal sentido Sus normas protegen valores esenciales compartidos por la comunidad internacional. Se puede decir que el lus Cogens es la encarnación jurídica de la conciencia moral de la sociedad internacional.

Es el artículo 53 de la Convención de Viena sobre Derecho de los Tratados (1969), el que define el lus Cogens como el conjunto de normas imperativas de derecho internacional general, establecidas por la comunidad internacional de Estados en su conjunto. Las normas de lus Cogens no pueden ser derogadas, salvo por otra norma del mismo rango. Cualquier tratado internacional contrario a una norma de lus Cogens es nulo.

\section{La perspectiva de la filiación y su relación con la educación inclusiva. Panorama actual en América Latina y Colombia}

A nivel de América Latina y Colombia la singularidad se convierte en el foco de atención de los procesos de inclusión educativa, ya que a través de esta se valida la identidad de cada uno y se favorece el bienestar, por lo tanto, la comprensión de la escuela como un espacio de inclusión permite el reconocimiento de las diferencias personales y las convierte en recursos para el aprendizaje, de manera que los estudiantes se sienten respetados, queridos y participes de los procesos que allí se gestan. Así, se contribuye en la construcción de una identidad personal y colectiva; personal en la medida en que el estudiante empieza a presentar y enriquecer una identidad y un proyecto de vida. Colectiva en el sentido de que se promueven valores como la equidad y la dignidad dentro de las escuelas. Como se puede apreciar, la inclusión implica un cambio para las escuelas hacia procesos que validen, apoyen y utilicen la singularidad de los estudiantes como un recurso. Pero para hacerlo, es 
necesario que se asuma la tarea, de identificar y remover, en su caso, las barreras que, desde distintos planos de la vida escolar, al interactuar negativamente con las condiciones personales o sociales de los alumnos en determinados momentos, pudieran limitar en cada centro, precisamente, su presencia, aprendizaje o participación.

La política pública que actualmente se plantea para Colombia en materia de educación inclusiva, retoma los postulados que hacen referencia a los principios de un Estado de derechos que reafirme la participación y fiscalización del conjunto social, concebida como un proceso en el cual la idea de la educación como derecho, de acuerdo con lo expresado por Valenciano Canett (2009) que va de lo jurídico a lo social en estos términos:

1. Toda persona tiene derecho a la educación. La educación debe ser gratuita, al menos en lo concerniente a la instrucción elemental y fundamental. La instrucción elemental será obligatoria. La instrucción técnica y profesional habrá de ser generalizada; el acceso a los estudios superiores será igual para todos, en función de los méritos respectivos.

2. La educación tendrá por objeto el pleno desarrollo de la personalidad humana y el fortalecimiento del respeto a los derechos humanos y a las libertades fundamentales; favorecerá la comprensión, la tolerancia y la amistad entre todas las naciones y todos los grupos étnicos o religiosos, y promoverá el desarrollo de las actividades de las Naciones Unidas para el mantenimiento de la paz.

3. Los padres tendrán derecho preferente a escoger el tipo de educación que habrá de darse a sus hijos (p. 14).

Como parte de este cambio necesario y de la superación de barreras, es indispensable que se realice una modificación en la forma como los adultos 
comprenden a los niños y adolescentes. Para Lansdown (2005), la tarea de los adultos es incrementar y apoyar el desarrollo de las facultades y características personales, y para hacerlo se deben respetar las capacidades de los niños para definir sus propios intereses y prioridades, así como las estrategias para abordarlos. También es importante que se logre cooperación entre los niños y entre estos y los adultos.

Para que los procesos de inclusión se favorezcan y se valide la singularidad de los estudiantes, es necesario que los maestros generen ciertos cambios al interior del aula. Estos cambios deben centrarse en generar un clima inclusivo donde todos se sientan acogidos y valorados; y el desarrollo de estrategias didácticas que garanticen el aprendizaje y participación de todos y cada uno de los estudiantes, de acuerdo con lo planteado por Figueroa Ángel, Gutiérrez de Piñeres Botero \& Velázquez León (2017), quienes hace alusión a lo propuesto por Moliner (2008) en el sentido que se deben "dar a conocer las singularidades individuales y en el caso en que no surja un apoyo entre iguales de forma espontánea, planificar la enseñanza de las competencias o habilidades necesarias para ello" (p. 19), desde luego, de acuerdo con el tiempo y al tipo de interacción que tienen los maestros y los estudiantes.

Sin embargo, estas iniciativas no pueden limitarse a un maestro o aula en particular, sino que debe ser la escuela, en su conjunto, la que articule respuestas coherentes y globales a los retos que presenta la inclusión y que evalúe en qué medida los valores, los principios, las normas, las relaciones, las costumbres y las formas de participación que se gestan al interior de la escuela pueden llegar a facilitar u obstaculizar la inclusión social. De igual manera, esas acciones involucran a las familias, las comunidades y el Estado, los cuales deben empezar a generar iniciativas de inclusión a nivel local y general, en donde la comprensión de la singularidad sea un valor que identifique a toda la población y que se promueva en todos los ámbitos nacionales. 


\section{Descripción del Sistema Educativo de Colombia en relación con los mecanismos de inclusión}

Con el actual sistema educativo de Colombia en el que el alumno debe amoldarse a la institución, se debe cambiar totalmente este pensamiento y la institución es la que debe adaptarse a las necesidades de cada uno de sus alumnos en este caso aquellos que necesitan una inclusión más que social, educativa.

Se debe también cambiar el perfil y el rol del docente, así como apoyar su desarrollo profesional lograr la inclusión supone, entre otras cosas, trabajar articuladamente en torno al tipo de docente deseado, jerarquizar su rol en atender las diversidades y el permanente apoyo a su trabajo en el aula.

Actualmente se presentan estas tres situaciones, las cuales son descritas y analizadas por Opertti (2015) en la siguiente forma:

(a) La constatación de la existencia de dos órdenes complementarios de problemas: (i) fuerte resistencia de los docentes a trabajar en escuelas y aulas heterogéneas e (ii) insuficiencia de competencias y conocimientos sobre cómo abordar las diversidades en los perfiles y en las maneras de aprender de alumnas y de alumnos.

(b) El predominio del discurso de la desviación que establece jerarquías situando a cada estudiante según sus competencias cognitivas por sobre el enfoque inclusivo que enfatiza el potencial de aprendizaje de cada estudiante que debe ser descubierto y estimulado de manera progresiva.

(c) Los currículums de formación docente carecen en general de un marco conceptual y de los instrumentos metodológicos para hacer frente a la diversidad de expectativas y necesidades de los educandos. 
(d) Compartir prácticas que parecen funcionar bien en diferentes ciudades o regiones - trabajo interdisciplinario en equipos docentes; apoyo a los docentes en escuelas comunes en relación a los estudiantes con necesidades especiales; la consideración de las escuelas especiales como centros de recursos; tutorías que impliquen un apoyo pedagógico personalizado; redes de contacto entre escuelas (por ejemplo, comunidades de prácticas docentes) y coordinación estrecha con instituciones sociales (concepción y gestión de políticas sociales). (p.p. 8-9)

\section{Descripción del Sistema Educativo en el Caribe Colombiano}

El sistema educativo en la Región del Caribe colombiano implicaba un proceso que en forma racional permitiese dar respuestas a la gran diversidad de las necesidades sentidas por todos los estudiantes canalizadas mediante estudios e investigaciones realizadas a través "de prácticas en las escuelas, las culturas y las comunidades reduciendo así la exclusión dentro de la educación, sintiendo la diversidad como un rasgo positivo del colectivo y logrando a través de las prácticas inclusivas un enriquecimiento en todos los sujetos implicados" (Mora Mora, 2010).

De acuerdo con los planteamientos de Valencia (2016) quien estableció que además de lo propuesto por Mora Mora también se hacía imperioso contar con lo siguiente:

(...) cambios y modificaciones en el contenido, los enfoques, las estructuras y las estrategias de la enseñanza, con una visión común que alcance a todos los niños de la misma edad y con la convicción de que es responsabilidad del sistema educativo ordinario educar a todos los niños, dándoles las mismas oportunidades de calidad y gratuidad (p.15). 
Son muchas las dificultades a las que se ha enfrentado la Región Caribe colombiana a lo largo de los años y en la actualidad. Sin embargo, cobra presencia el considerar que frente a la situación de implementar un nuevo régimen educacional para mejorar la calidad estudiantil del territorio faltaría en forma decidida que exista mayor acompañamiento y seguimiento en cuanto a las nuevas implementaciones de las Organizaciones sin Ánimo de Lucro para que se sumen al cumplimiento de los actores del sistema educacional con la intención de mejorar la educación en el Caribe colombiano con más fuerza y compromiso.

En los últimos años, las estadísticas educativas de Colombia han mejorado: entre los años 2002 y 2005, el número de estudiantes de educación básica y media en el país aumentó en 800.000 , hasta llegar a cerca de 11 millones de estudiantes, destacándose entre otros aspectos, que a nivel regional, los estudiantes en la Costa Caribe aumentaron en 300.000, llegando a 2,7 millones. Durante el mismo período, la cobertura regional y nacional en educación básica pasó de $82 \%$ a $88 \%$. Otro de los indicadores que muestra el crecimiento del sistema educativo colombiano es la tasa de analfabetismo, la cual disminuyó en 4,5 puntos porcentuales entre 1990 y 2005.

En cuanto a la calidad de la educación, es indispensable medirla periódicamente en sus tres niveles (primaria, secundaria y superior), así como difundir los logros esperados en cada uno de ellos, para que la sociedad y los padres de familia conozcan el comportamiento del sector y escojan con suficiente información el colegio o universidad para sus hijos.

En Colombia se practican varios exámenes a los estudiantes como las pruebas Saber, los exámenes al terminar la educación básica (exámenes del lcfes) y las pruebas Ecaes (al terminar la educación superior). Con respecto a estas pruebas es preocupante saber que en la mayoría de las ocasiones los resultados de los estudiantes costeños están por debajo de la media nacional. 
Una política de desarrollo regional para el Caribe colombiano, necesita acciones encaminadas a elevar el nivel de su recurso humano, como una forma de superar su rezago económico y mejorar la calidad de vida de su población. Es oportuno analizar la experiencia de la Secretaría de Educación de Bogotá con sus Programas de 'Banco de Oferentes' y convenios de concesión con instituciones educativas privadas.

Si bien es cierto que en algunas instituciones educativas del Caribe colombiano los Modelos Pedagógicos que se aplican en la práctica no incluyen a las personas que tienen algunas limitaciones de carácter cognoscitivo o físico aunque las disposiciones reguladas por el Decreto 366 del 2009 (Ministerio de Educación Nacional, 2009), "por medio del cual se reglamenta la organización del servicio de apoyo pedagógico para la atención de los estudiantes con discapacidad y con capacidades o con talentos excepcionales, en el marco de la educación inclusiva" (p.1), son específicas y perentorias al respecto. Sin embargo, se pregona que existen excepciones en algunos lugares de la Región, que están mostrando que los niveles de calidad en cuanto a la inclusión de la población son adecuados, de acuerdo con un estudio realizado en la ciudad de Cartagena por García Mejía (2011) y un grupo de estudiantes de la Corporación Universitaria Rafael Núñez.

\section{Las estrategias implementadas como garantías para una educación inclusiva desde la filiación en Colombia}

Como estrategia, el Ministerio de Educación Nacional para el logro de la inclusión educativa, se ha fomentado la idea de brindar apoyo a las entidades territoriales para que, a través de las Secretarías de Educación, logren avanzar en sus procesos de reorganización, asignación de docentes, coordinación de acciones necesarias para identificar y caracterizar las poblaciones vulnerables dentro y fuera del sistema; proponer orientaciones pedagógicas para elaborar y adecuar currículos; implementar, identificar y desarrollar modelos educativos flexibles; crear herramientas pedagógicas 
y didácticas; definir canastas educativas, formar a funcionarios, directivos docentes y docentes, entre otros.

Para el desarrollo de estrategias pedagógicas las entidades territoriales deben tener en cuenta: Utilizar modelos educativos flexibles para la atención etno-educativa; permitir la flexibilidad curricular; promover el intercambio de experiencias pedagógicas, para mejorar los procesos educativos; e impulsar el desarrollo de nuevos modelos acordes con las necesidades de la población que será atendida; promover la capacitación de etno-educadores; realizar un inventario de instituciones y centros educativos que atienden poblaciones étnicas, así como de los programas y proyectos de etno-educación; delimitar las estrategias institucionales, pedagógicas y financieras, definiendo las metas de corto, mediano y largo plazo.

Por otro lado, para mejorar la pertinencia y calidad de prestación del servicio educativo a las poblaciones vulnerables y combatir la deserción se han implementado modelos de educación sugeridos por la Comunidad de Práctica en Desarrollo Curricular Región Andina /OIE-UNESCO (2007) flexibles, acompañados de herramientas pedagógicas y didácticas, canastas educativas y formación de funcionarios, directivos docentes y docentes.

\section{El panorama de la educación en Colombia con inclusión}

Los recursos asignados para la educación casi siempre son producidos después de la súplica; aparentemente, la calidad de la educación es ajena a lo público y hace falta un mayor compromiso del Estado para garantizarla a los niños y niñas menos favorecidas económicamente; ellos y ellas también deberían tener la posibilidad de pensarse como grandes personajes del país.

El acceso, porque a pesar de una política educativa que permite la integración y que se evidencia en la Constitución Política de Colombia (1991), la Ley 115 (1994) y el Decreto 2082 (1996), la integración al aula es información desconocida por los padres 
y por este motivo, no hacen válido el derecho que tienen los niños y las niñas a una integración a la escuela regular. Hace falta conocimiento, promoción y sensibilización sobre lineamientos de política que facilitan que los estudiantes realmente ingresen.

La evaluación masiva reglamentada en los estándares y las competencias que orientan los procesos educativos en Colombia, excluyen la población con discapacidad. Los procesos pedagógicos y de evaluación llevan a la inequidad, debido a que no se tiene en cuenta las diferencias. En otros casos, no es una cuestión simplemente de normatividad y mucho menos de tolerancia. Lo importante es que la sociedad piense en el sentido mismo de la vida, preguntándose el para qué de la vida. Un país incluyente se da a partir de opciones que genera la sociedad en su conjunto permitiendo la convivencia de todos. Colombia, es un país atrasado en procesos de inclusión; se ha avanzado muy poco en la reflexión sobre el significado de la palabra.

Con el Decreto 2082 (1996), inicialmente los estudiantes fueron ubicados físicamente en las aulas. Al estar allí, se generó dentro del aula un análisis sobre cómo trabajar con estos niños. Se pensó entonces en la necesidad de capacitar a los docentes y de buscar profesionales de apoyo. Si bien es cierto que hubo un cambio de paradigma que permitió dejar de ver la discapacidad como enfermedad, como algo individual, para verla como algo social donde la comunidad educativa tiene que responder y pensarse, se mira la institución de otra manera en cuanto a los procesos de inclusión que en otros países está muy avanzado y que aquí hasta ahora se ha visto como integración. Si se logra ver la dimensión y el significado de la inclusión, se dará paso a adaptaciones curriculares e institucionales, para las cuales las instituciones no están aún preparadas.

En definitiva, hay tres factores que no se han trabajado y el peso de ese compromiso, recae directamente sobre las instituciones educativas, sobre los maestros y sobre la comunidad: la cultura (porque la responsabilidad no recae solo sobre los maestros; no son ellos los únicos en tener contacto con poblaciones vulnerables); la 
política (si se hace parte de la formulación de políticas, se es reconocido); y las prácticas pedagógicas (el constante trabajo de formar para la vida).

La Corte Constitucional colombiana se ha manifestado jurisprudencialmente en varias ocasiones acerca de la educación inclusiva desde la perspectiva de la filiación, con las siguientes providencias las cuales se mencionan a continuación:

- Sentencia T-679/16. Los niños y niñas son titulares del derecho fundamental a la educación en condiciones de igualdad, independientemente de las limitaciones físicas, cognitivas o de cualquier otro tipo que presenten. De este modo, ante una situación que genere discapacidad, el Estado debe eliminar las barreras que impidan el goce y disfrute efectivo de esa garantía, a través de la inclusión en el sistema tradicional, o en el especializado cuando las circunstancias lo ameriten, por ejemplo, cuando sea imposible garantizar la disponibilidad, el acceso, la aceptabilidad, la permanencia o la adaptabilidad (Corte Constitucional, 2016).

- Sentencia T-850/14. Frente al derecho a la educación de las personas con discapacidad, y con fundamento en el valor y principio a la igualdad material, esta Corporación ha reconocido que son personas, capaces de gozar plenamente de todos sus derechos fundamentales entre los cuales se encuentra el derecho a la educación, Razón por la cual, corresponde al Estado garantizar el goce efectivo de todos sus derecho, en las condiciones más favorables posibles. Cuando se trata de personas en situación de discapacidad, la educación debe prestarse en condiciones de igualdad, atendiendo las particularidades de cada caso, de tal forma que el proceso de aprendizaje se adapte a sus condiciones y en este sentido pueda acceder al mismo como cualquier persona, es decir, que a estas personas se les debe garantizar una educación inclusiva, que consiste en ampliar el espectro de inclusión de personas con necesidades educativas especiales, más allá del acceso a la escuela regular (Corte Constitucional, 2014). 
- Sentencia T-318/14. El artículo 67 de la Constitución establece la obligación del Estado de asegurar a los niños las condiciones necesarias para su acceso y permanencia en el sistema educativo, deber que adquiere especial relevancia cuando se trata de personas con limitaciones físicas o mentales, conforme a los artículos 47 y 68 ídem, pues en tales eventos concurren en ellas dos circunstancias de vulnerabilidad que requieren de acciones de especial protección en materia educativa: i) son menores de edad, es decir, personas en proceso de formación y desarrollo; y ii) la condición de limitación o discapacidad (Corte Constitucional, 2014). En este sentido, el artículo 47 de la Constitución Política de Colombia (1991) impone al Estado adelantar una política de integración social para los disminuidos físicos, sensoriales y psíquicos, a quienes se prestará la atención especializada que requieran, proceso a favor del cual se establecen medidas de inclusión escolar.

- Sentencia T-523/16. La educación inclusiva es una apuesta por reconocer la diversidad y la dignidad humana, a través de un modelo en el que concurren en el aula personas con diferentes capacidades para acceder a ciertos conocimientos y potenciar sus habilidades. Se fundamenta en que todas las personas deben compartir los mismos espacios y no pueden ser apartadas en razón de determinadas características que tradicionalmente han sido catalogadas como limitaciones para recibir la misma educación. La educación inclusiva reconoce las diferencias entre todas las personas y destaca la igualdad que debe prevalecer en la sociedad. Se aparta de la diferenciación de normalidad y anormalidad, que se constituía en una categorización para juzgar y aislar a un grupo y restringirle desde el comienzo de la vida, sus derechos (Corte Constitucional, 2016). 


\section{Las directrices del Ministerio de Educación Nacional - MEN con respecto a los Modelos Pedagógicos que se implementan en Colombia. Un análisis retrospectivo}

Como complemento, el MEN forma a los maestros en temáticas relacionadas con herramientas pedagógicas y desarrollo de competencias para la convivencia. Los talleres de herramientas pedagógicas se realizan por ciclos de capacitación que comprenden las siguientes temáticas:

- Derechos humanos: al abordar esta temática, se capacita a los docentes en temas relacionados con los derechos de los niños y las niñas, de manera que la educación sea un derecho fundamental que debe restituirse con todas las garantías a las poblaciones vulnerables y a los niños en general.

- Cultura de paz: dada la experiencia traumática por la que han atravesado los niños, niñas y jóvenes en situación de desplazamiento, se hizo necesario brindar herramientas a los docentes que les permitieran identificar la fuente de los conflictos de los estudiantes y contar con mecanismos que contribuyan a su solución dentro y fuera del aula.

- Salud sexual y reproductiva: la población en situación de desplazamiento presenta otras dificultades asociadas, como son el embarazo precoz y la poca información acerca del manejo de su sexualidad. En este sentido, se buscó brindar herramientas a los docentes para trabajar con los niños, niñas y jóvenes a fin de hacerles conocer sus derechos sexuales y reproductivos.

- Herramientas pedagógicas: les permiten a los docentes cualificar su rol a través de la identificación de elementos de análisis e intervención pedagógica para niños, niñas y jóvenes afectados por la violencia, con el fin de mejorar sus procesos de enseñanza aprendizaje. 
- Competencias ciudadanas: este ciclo de talleres comprende temáticas que permiten promover el desarrollo de competencias sociales en sus estudiantes; es decir, la capacidad o habilidad para interactuar de manera exitosa con su ambiente y lograr la satisfacción legítima de sus necesidades, logrando en ellos actitudes de confianza, autonomía y responsabilidad. De igual manera, promueve el desarrollo de competencias para la solución y regulación de los conflictos en sus estudiantes; es decir, identifica conflictos y enseña a los estudiantes a resolverlos cuando se presentan en el aula y fuera de ella.

- Competencias personales: lograr en los estudiantes la superación de las secuelas tras la experiencia de la violencia. Estas competencias personales incluyen aspectos motivacionales, afectivos y actitudinales que permiten en los estudiantes la interiorización como un valor de los derechos humanos. El Decreto 2562 (2001), por el cual se reglamenta la Ley 387 (1997), establece que las entidades territoriales deben: Desarrollar programas de formación y capacitación a docentes que atienden la población desplazada y por otro lado, el Decreto 250 del 2005, el cual deroga el Decreto 173 de 1998, establece que el sector educativo debe mejorar la calidad de la educación mediante el desarrollo de planes y programas de capacitación de docentes, que optimicen los procesos para la atención a esta población.

\section{Muestras significativas de la práctica inclusiva en el sistema educativo del Caribe colombiano. Hacia el desarrollo de estrategias para la Educación inclusiva desde la perspectiva socio-jurídica de la filiación, en el Caribe colombiano}

El conocimiento que se tiene sobre los estudiantes y sus necesidades, además de proporcionar información relacionada con las diferencias significativas desde lo social, económico, cultural, etcétera, constituyen los aspectos fundamentales que se van transmitiendo de una generación a otra, como parte de los contrastes entre las comunidades de una misma Región entre sí, de manera particular y en términos general de una sociedad global. El conocimiento de estas diferencias significativas es lo que 
posibilita el lograr una educación inclusiva en la cada una de los departamentos de la Región Caribe colombiana, y también en el país. Entre otros, algunos de estos conocimientos son necesarios e indispensables a tener en cuenta para tomar mejores decisiones con las cuales se procuren implementar decisiones:

- Conocer a tus alumnos. Los maestros necesitan conocer a sus alumnos y este es el paso número uno para entender las necesidades de cada estudiante. Conocer a los estudiantes es una forma de entender su mirada, su contexto, sus dificultades y sus fortalezas y, por ende, es una estrategia clave para crear espacios que apuntan hacia la inclusión. Todos los estudiantes son diferentes y entenderlos es fundamental para cumplir esta meta.

- Transmitir y creer. A lo largo del tiempo se nos ha enseñado que ser diferente es algo malo, ¿qué debemos ser "normales" pero si todos somos seres únicos y diferentes que vendría siendo normal?, es mejor entender las diferencias y aceptarlas como parte del enriquecimiento del aula. Pero más importante aún es creer realmente en esto y saber transmitir los aspectos positivos de dichas diferencias para que los mismos estudiantes saquen provecho de esto y entiendan que no hay nada de malo en ello.

- La comunicación. No sólo es necesario conocer a todos los estudiantes, también es importante mantener una comunicación constante y efectiva con todos. Para eso, se deben buscar espacios de diálogo que permitan entender aún más sus necesidades, impresiones, preocupaciones y opiniones. Ellos son los que más pueden ayudar a la hora de medir las dinámicas del trabajo inclusivo.

- Las familias. Conocer a los estudiantes también significa conocer a sus familias, el entorno donde conviven aparte de la escuela, con quienes se relacionan fuera de ella. Ellos son una pieza clave del aprendizaje y un recurso muy valioso al pensar en un aula inclusiva. Forjar relaciones de confianza con ellos ya que son una parte esencial en la vida de los alumnos. 
Con ocasión a este aspecto Viloria (2008) lo había destacado en una publicación, recalcando que la Región Caribe de Colombia necesita de acciones concretas y decisivas para disminuir la brecha en materia educativa con otras regiones del país, en los siguientes términos:

Una política de desarrollo regional para el Caribe colombiano, necesita acciones encaminadas a elevar el nivel de su recurso humano, como una forma de superar su rezago económico y mejorar la calidad de vida de su población. Es oportuno analizar la experiencia de la Secretaría de Educación de Bogotá con sus Programas de 'Banco de Oferentes' y convenios de concesión con instituciones educativas privadas. (...) El gran esfuerzo de la política educativa en el Caribe colombiano debería centrarse en universalizar la cobertura de secundaria y expandir la de preescolar, así como fortalecer los programas que atiendan a la población más pobre y vulnerable de la región. Una educación de preescolar de buena calidad, puede aumentar la permanencia en el sistema y tener efectos positivos sobre los resultados académicos posteriores.

Así mismo, para mejorar la calidad de la educación se requiere que los gobiernos impulsen una política de capacitación y formación continua de docentes; que los maestros se organicen en torno a comunidades académicas (y no sólo gremiales) y que los rectores ejerzan un fuerte liderazgo sobre la organización escolar. Esto implica un real compromiso de las instituciones costeñas, sus dirigentes, estudiantes y maestros; es el reto que debemos asumir para no continuar ampliando la brecha que existe entre la educación de la región y la del resto del país (p. 2). 


\section{Descripción del Sistema Educativo en la ciudad de Barranquilla}

La Educación en la ciudad es regulada por la Secretaría de Educación, dependencia de la Alcaldía Distrital. La ciudad ofrece el sistema educativo nacional gratuito en sus niveles de primaria y secundaria. A nivel superior, Barranquilla se distingue por ser el principal centro universitario regional de la Costa Caribe; también se puede acceder a formación técnica y tecnológica. Entre las décadas de 1960 y 1990, la ciudad sirvió de receptora de la población estudiantil desatendida de otras regiones de la Costa y algunas del resto del país, que no podían cursar estudios de educación superior a falta de instituciones en sus lugares de origen.

La educación pública en la capital del departamento del Atlántico ha venido avanzando a grandes pasos en los últimos años, con un hecho desprendido de la apuesta implementada desde la administración distrital para garantizar este derecho a los jóvenes y niños en esta parte del país. En la actualidad el Distrito de Barranquilla cuenta con 154 instituciones educativas.

Para garantizar la calidad en la educación se han invertido más de 20.000 millones de pesos y actualmente se cuentan con 27 programas en las instituciones educativas para brindarles acompañamiento y reforzar los conocimientos de los estudiantes y docentes. Uno de los programas es Tu Ruta a la U, implementado por la Secretaría de Educación distrital en compañía con la Universidad del Norte para el fortalecimiento académico de 8 mil estudiantes de grado 11 de las 154 instituciones educativas distritales en las pruebas Saber 11, y brindar acceso a la educación superior.

Este proyecto está orientado a que durante cinco meses se brinden refuerzos escolares enfocados en los diferentes componentes de la prueba Saber cómo las matemáticas y razonamiento cuantitativo, ciencias sociales y competencias ciudadanas, biología, química, física e inglés. El programa contiene además formación para más de 500 docentes por institución de las áreas básicas, para acompañar y dar seguimiento al proceso de formación que llevan los estudiantes. 
Otros de los programas académicos en los que se ha invertido desde el distrito es en la jornada única, que busca garantizar el mejoramiento de la calidad educativa mediante el incremento del tiempo de los estudiantes en las IED para el fortalecimiento de las competencias básicas y ciudadanas, como la reducción de los factores de riesgo y vulnerabilidad a los que se encuentran expuestos los estudiantes en su tiempo libre.

La educación inclusiva desde la perspectiva socio jurídica en la ciudad de Barranquilla se fundamenta en la expectativa de acoger a todos los estudiantes, independientemente de sus características personales, culturales, cognitivas entre otras. Para ello su entorno educativo debe ofrecer condiciones de accesibilidad para todos, desarrollando estrategias organizativas con respuestas eficaces para abordar la diversidad.

Con relación a esta situación, reiteradamente se dice que: La inclusión es sinónimo de integración; la inclusión amplía la integración; la inclusión es un reto educativo; todos iguales, todos diferentes; la adaptación de la enseñanza es para todos los estudiantes y se debe dar atención a todos dentro del aula ordinaria, independientemente de las condiciones de discapacidad que presenten algunos estudiantes.

Figueroa Ángel, Gutiérrez de Piñeres Botero \& Velázquez León (2017) precisaron e hicieron saber que la inclusión se genera como oposición a la exclusión social y hace referencia "a las personas con discapacidad mental o física, padres solteros, toxicómanos, entre otros individuos sin seguridad social" (p. 14). Esta conceptualización se ajusta a lo que se vive en Colombia y el Distrito de Barranquilla. 


\section{La implementación y desarrollo de estrategias para la Educación en la ciudad de Barranquilla}

El desarrollo cognoscitivo es un proceso que implica habilidades que van adquiriéndose desde el momento mismo del nacimiento hasta los diferentes instantes en que el individuo ejecuta actividades correspondientes a cada nivel de desarrollo, con mayor destreza. Es vital comprender cuáles son y cómo se están adquiriendo las habilidades que explican parcialmente no sólo el desarrollo cognoscitivo sino la predicción de las formas posteriores de aprendizaje y, por ende, de éxito académico. De acuerdo con ello, las estrategias cognoscitivas son habilidades inter-organizadas cuya función es regular y verificar el uso de conceptos y reglas y a medida que los individuos desarrollan distintos tipos de habilidades intelectuales, a lo largo de su proceso de aprendizaje se producen también cierto tipo de procesos a nivel interno que les permiten aprender y almacenar diferentes habilidades que sirven como mecanismos para la autorregulación de los procesos asociados con el aprendizaje.

En otras palabras, los individuos van aprendiendo a aprender, es decir, van aprendiendo cómo efectuar el razonamiento analítico y reflexivo que conduce a un mayor aprendizaje. Es evidente que conforme el individuo sigue aprendiendo aumenta su capacidad autodidáctica, o incluso lo que podría llamarse aprendizaje independiente. Esto debido a que va adquiriendo estrategias cada vez más eficaces para regular sus propios procesos internos.

Con el objetivo de seguir trabajando en equipo por la calidad educativa de Barranquilla, con relación a uno de los propósitos de la Alcaldía Distrital, la Secretaría de Educación realizó un encuentro con los rectores de los colegios privados de la ciudad, con la perspectiva de trazar nuevos objetivos y construir acuerdos con todos los actores educativos (directivas, docentes, padres de familia, estudiantes y comunidad empresarial vecina) para fortalecer las instituciones y las relaciones escolares, las cuales redundarían en la calidad de la educación. 
Como un espacio de reflexión y planificación, se realizó el evento donde asistieron 208 rectores de instituciones educativas públicas y privadas, con quienes se analizaron en primera instancia, los resultados que se muestran en el Índice Sintético de Calidad Educativa (ISCE) y los avances en el Mejoramiento Mínimo Anual (MMA), con el fin de fortalecer estrategias y nuevas metas. Durante la jornada, la Secretaría de Educación, manifestó lo siguiente:

$\mathrm{Si}$ en algo hemos marcado la diferencia es que en Barranquilla estamos trabajando unidos para formar a los mejores ciudadanos del país. Por eso abrimos estos espacios de reflexión, para que juntos soñemos en grande la mejor educación de nuestros niños y jóvenes. Aquí le estamos poniendo corazón, conocimiento y pasión a estos objetivos (Zona Cero, 2017).

Según los resultados en el Índice Sintético de Calidad Educativa (ISCE) en la ciudad, los colegios privados aumentaron el puntaje global en primaria de 6,33 a 6,67; en secundaria de 6,25 a 6,44 y en la media de 6,2 a 6,45. Entre los colegios que se destacaron por su mejoramiento en el ISCE se encuentra el Instituto Colombiano de Educación para el Desarrollo Humano Especializado- ICETEC- con 9,18 en básica primaria y 8,67 en básica secundaria, mientras que en la media se destaca el Colegio Colón con un puntaje de 9,58.

En la jornada, los rectores de las instituciones educativas participaron de un espacio de análisis, retroalimentación y reflexión, hicieron una autoevaluación institucional para saber cómo se pueden fortalecer y obtener mejores resultados, además de la proyección de nuevas metas en cada colegio (Zona Cero, 2017).

En las IED del Distrito de Barranquilla que estuvieron vinculadas al estudio, cuando se realizó la indagación sobre las estrategias y actividades implementadas para 
la educación inclusiva, se encontró a través de sus respuestas en las entrevistas y por observación ${ }^{4}$ que:

- Cada profesor implementa la educación inclusiva para que ningún estudiante se sienta discriminado.

- En las IED se siguen los lineamientos del Ministerio de Educación.

- Se esfuerzan por incluir a los estudiantes en las actividades educativas.

- Los docentes han asimilado el rol de ser incluyentes.

- Utilizan materiales didácticos y medios audio visuales en algunas de las instituciones observadas para la mayor comprensión de los niños.

- Dan a conocer a los estudiantes la importancia de la no discriminación por raza, sexo, religión, etcétera.

- Los docentes realizan intercambios con los estudiantes en todas las actividades. Ello también incluye a los que presentan discapacidades diferentes o excepcionales.

- Hacen uso de las estrategias propositiva, argumentativa, comprensiva, con talleres en el aula de clases.

- Han intentado adecuar el curriculum a las necesidades del estudiante ${ }^{5}$.

\footnotetext{
${ }^{4}$ Observación a Instituciones Educativas Distritales IED de Barranquilla y entrevistas con docentes. Barranquilla, mayo-noviembre de 2018.

${ }^{5}$ Observación a Instituciones Educativas Distritales IED de Barranquilla y entrevistas con docentes. Barranquilla, mayo-noviembre de 2018.
} 
Por otra parte, la Secretaría de Educación del Distrito de Barranquilla (2017) ha propuesto una serie de actividades que refuerzan la aplicación de la Educación inclusiva, como las siguientes:

- Un Programa para la atención de la población con necesidades educativas especiales.

- La realización de encuesta para obtener información diagnóstica de los docentes de Barranquilla.

- Indagación en los docentes para conocer de ellos sobre sus necesidades de formación (Especialización, Maestrías y Doctorados).

\section{Algunas propuestas de Educación Inclusiva en ejecución en los IED de Barranquilla}

Es evidente que conforme el individuo sigue aprendiendo aumenta su capacidad autodidáctica, o incluso lo que podría llamarse aprendizaje independiente. Esto debido a que va adquiriendo estrategias cada vez más eficaces para regular sus propios procesos internos.

Algunas de las propuestas que se pueden considerar destacadas fueron las presentadas por las Instituciones Educativas Distritales como el Colegio Salvador Suárez Suárez que ha implementado estrategias de atención a la población sorda; Colegio Nuestra Señora del Carmen, que ha diseñado una nueva infraestructura tecnológica necesaria para atender a niños y niñas especiales.

\section{Conclusiones}

A pesar del esmero y la dedicación con que las diferentes IED quieren implementar la educación inclusiva, se advierte en los docentes y en el ambiente escolar que ello se hace por que existe una imposición por parte del Ministerio de Educación y no porque sea una condición natural de cada educador o la filosofía de las 
instituciones educativas. En el seno del mismo Ministerio de Educación se encuentran los principales obstáculos que se convierten en una limitante para que la inclusión no sea efectiva ni eficaz.

La cultura de la exclusión se ha hecho notoria en la educación y ella socava el sistema educativo no dándoles oportunidades a las familias a gozar de ambientes escolares posibilitadores para que sus hijos que padecen cualquiera de las limitantes que han sido descritas anteriormente, puedan encontrar un clima educativo adecuado, que definitivamente los cobije y se pueda logar la inclusión de la que actualmente se pregona en todo el territorio colombiano.

También se advierte resistencia por parte de los directivos de las IED, los docentes, los padres de familia y en algunos casos, los mismos estudiantes quienes portan un logotipo de la exclusión desde todos los ámbitos en los espacios escolares Estas situaciones se encuentran en el interior y en las relaciones cotidianas de los hogares y en las comunidades donde impera la exclusión a quienes se encuentran en diferente posición con respecto a los demás por su condición de vulnerabilidad.

Ahora que Colombia ha tenido que enfrentar uno de los fenómenos poblacionales que ha sacudido los cimientos de la estructura de todos los sectores, el sistema educativo se enfrenta a su más dura realidad: atender población local y población migrante en educación. En tal sentido, la sociedad colombiana en el sector educativo ha sido desbordada por las exigencias y las solicitudes de atención por parte de la población migrante que exige atención y en cada una de las instituciones educativas la respuesta ha estado limitada por la misma crisis que aborda al sector educación. Crisis que se observa incluso en los particulares en sus diferentes roles, padres, hijos, servidores públicos, consumidores (Álvarez, 2009), estudiante mismo.

En educación la inclusión no se debe cumplir por la existencia de una ley o un decreto. Debe ser una condición natural de cada persona. 


\section{Referencias}

Alvarez Estrada, J. (2009) (1). La libertad contractual del consumidor en Colombia: ¿mito o realidad? contratos de adhesión condiciones generales del contratocláusulas abusivas. Erg@omnes, 1(1), 136-165. https://doi.org/https://doi.org/10.22519/22157379.241

Asamblea Nacional Constituyente. (2010). Constitución Política de Colombia 1991. Bogotá: Consejo Superior de la Judicatura. Disponible en: https://www.ramajudicial.gov.co/documents/10228/1547471/CONSTITUCION-Interiores.pdf

Berrocal Duran, J. (2012). Similitudes y diferencias entre modelos pedagógicos de las instituciones del Caribe Colombiano. Erg@omnes, 4(1), 1-15. https://doi.org/https://doi.org/10.22519/22157379.212

Congreso de la República de Colombia. (1997). Ley 387 (1997). Disponible en: https://www.unidadvictimas.gov.co/sites/default/files/documentosbiblioteca/ley-387-de-1997.pdf

Congreso de la República de Colombia. (1994). Ley 115 de 1994. Bogotá: Congreso de la República de Colombia. Disponible en:

https://www.mineducacion.gov.co/1621/articles-85906_archivo_pdf.pdf

Convención de Viena sobre el derecho de los tratados. (1969). Convención de Viena sobre el derecho de los tratados. U.N. Doc A/CONF.39/27 (1969), 1155 U.N.T.S. 331, entered into force January 27, 1980. Disponible en: https://www.oas.org/xxxivga/spanish/reference_docs/convencion_viena.pdf

Corte Constitucional. (2016). Sentencia T-679/16. Bogotá: Corte Constitucional. Disponible en: http://www.corteconstitucional.gov.co/relatoria/2016/T-679-16.htm

Corte Constitucional. (2016). Sentencia T-523/16. Bogotá: Corte Constitucional. Disponible en: http://www.corteconstitucional.gov.co/relatoria/2016/t-523-16.htm

Corte Constitucional. (2014). Sentencia T-850/14. Bogotá: Corte Constitucional. Disponible en: http://www.corteconstitucional.gov.co/relatoria/2014/t-850-14.htm

Corte Constitucional. (2014). Sentencia T-318/14. Bogotá: Corte Constitucional. Disponible en: http://www.corteconstitucional.gov.co/relatoria/2014/T-318-14.htm

Figueroa Ángel, M. X.; Gutiérrez de Piñeres Botero, C. \& Velázquez León, J. (2017). Estrategias de inclusión en contextos escolares. En: Revista Diversitas - 
Perspectiva Psicológica, Vol. 13 No. 1 (2017), pp. 13-26. Disponible en: http://www.scielo.org.co/pdf/dpp/v13n1/1794-9998-dpp-13-01-00013.pdf

Lansdown, G. (2005). Las implicaciones de la revolución de las facultades del niño para la realización de sus derechos. Roma: Unicef, Save the Children, Centro de investigaciones Innocenti.

Mora Mora, R. (2010). Biografías de Instituciones de la Región Caribe. Barranquilla: Ediciones Universidad Simón Bolívar.

Ministerio de Educación Nacional MEN. (2019). Colombia avanza hacia una educación inclusiva con calidad. En: Boletín del 15 de noviembre de 2018. Disponible en: https://www.mineducacion.gov.co/cvn/1665/article-168443.html

Ministerio de Educación Nacional. (2009). Decreto 366 de 2009. Disponible en: https://www.mineducacion.gov.co/1621/articles182816_archivo_pdf_decreto_366_febrero_9_2009.pdf

Ministerio del Interior. (1998). Decreto 173 de 1998. Disponible en: https://www.minsalud.gov.co/sites/rid/Lists/BibliotecaDigital/RIDE/DE/DIJ/Decreto -0173-de-1998.pdf

Ministerio de Educación Nacional. (1996). Decreto 2082 de 1996. Disponible en: https://www.mineducacion.gov.co/1759/articles-103323_archivo_pdf.pdf

Opertti, R. (2015). La Educación Inclusiva, perspectiva internacional y retos de futuro. En: United Nations Educational, Scientific and Cultural Organization / UNESCO. Disponible en: https://www.researchgate.net/profile/Renato_Opertti/publication/237301482_La_ Educacion_Inclusiva_perspectiva_internacional_y_retos_de_futuro/links $/ 56 \bar{b} 1 \mathrm{fe} 3$ 008aed7ba3fedac38/La-Educacion-Inclusiva-perspectiva-internacional-y-retosde-futuro.pdf?origin=publication_detail

Organización de las Naciones Unidas para la Educación, la Ciencia y la Cultura Unesco. (2009). Directrices sobre políticas de inclusión en la educación. Paris: Organización de las Naciones Unidas para la Educación, la Ciencia y la Cultura. Disponible en: http://unesdoc.unesco.org/images/0017/001778/177849s.pdf

Organización de las Naciones Unidas para la Educación, la Ciencia y la Cultura Unesco. (2007). Colombia. La dificultad para concretar las normas en inclusión educativa. Taller Regional Preparatorio sobre Educación Inclusiva. Disponible en:

http://www.ibe.unesco.org/fileadmin/user_upload/Inclusive_Education/Reports/bu enosaires_07/colombia_inclusion_07.pdf 
Presidencia de la República de Colombia. (2005). Decreto 250 de 2005. Disponible en: https://www.unidadvictimas.gov.co/sites/default/files/documentosbiblioteca/decret o-250-de-2005.pdf

Presidencia de la República de Colombia. (2001). Decreto 2562 de Noviembre 27 de 2001. Disponible en: https://www.mineducacion.gov.co/1759/articles86131_archivo_pdf.pdf

Presidencia de la República de Colombia (1996). Decreto 2082 de 1996. Bogotá: Presidencia de la República de Colombia (1996). Disponible en:

https://www.mineducacion.gov.co/1759/articles-103323_archivo_pdf.pdf

Secretaria Distrital de Educación. (2017). Distrito y colegios privados plantean estrategias para seguir mejorando en calidad educativa. Disponible en: http://educa.barranquilla.edu.co/index.php/servicios/noticias/735-distrito-ycolegios-privados-plantean-estrategias-para-seguir-mejorando-en-calidadeducativa.

Valencia, J. (2016). Elementos de discusión para la mejora de la calidad del servicio educativo en la Región Caribe colombiana: una mirada desde los indicadores. Disponible en:

https://www.uninorte.edu.co/web/blogunivoluntarios/inicio/-/blogs/colombia-elreto-de-la-educacion

Valenciano Canett, G. (2009). Construyendo un concepto de educación inclusiva: una

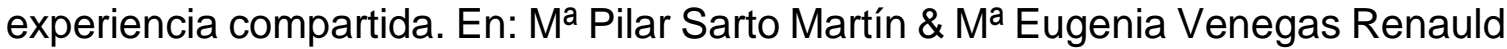
(Coordinadoras). Aspectos clave de la Educación Inclusiva. Salamanca: Publicaciones del INICO. Colección Investigación. Disponible en: http://sid.usal.es/idocs/F8/FDO22224/educacion-inclusiva.pdf

Viloria, J. (2008). Más y mejor educación para el Caribe colombiano. Disponible en: https://www.mineducacion.gov.co/observatorio/1722/article-141619.html

Zona Cero. (2017). Distrito y colegios privados, asociados para trabajar por la educación en Barranquilla. Disponible en: http://zonacero.com/generales/distritoy-colegios-privados-asociados-para-trabajar-por-la-educacion-en-barranquilla 